\title{
Fan Health Network: Feasibility, Acceptability and Preliminary Effects of a Healthy Lifestyle Behavior App to Improve University Faculty and Staff Well-being
}

\author{
Megan Amaya, PhD, CHES \\ Bernadette Melnyk, PhD, APRN-CNP, FAANP, FNAP, FAAN \\ Laurel Van Dromme, MA \\ The Ohio State University
}

\begin{abstract}
Background: Mobile health apps (mhealth) are designed to improve the health of patients through multiple functionalities, such as access to medical care services, wearable health tracking devices, personal digital assistants (PDA) and other portable and wearable network-capable gadgets.

Aim: The purpose of this pilot study was to test the feasibility, acceptability, and preliminary effects of the Fan Health Network (FHN) healthy lifestyle behavior application (app) on university faculty and staff's healthy lifestyle beliefs, healthy lifestyle behaviors, perceived stress and health outcomes.

Methods: A pre-experimental, one group pre-posttest design was used. Valid and reliable measures were used to assess healthy lifestyle beliefs, healthy behaviors, and perceived stress.

Results: Healthy behaviors and perceived stress significantly improved over the intervention time frame. Although not statistically significant, small improvements were seen in body mass index and waist circumference at the three month follow-up. Feasibility and acceptability were considered average on app usefulness, helpfulness, and motivational components.

Conclusion: The FHN app has potential as a key wellness intervention strategy for individual health promotion as well as for organizations, such as the workplace, to improve population health outcomes.
\end{abstract}

Submitted 3 September 2019: accepted 16 November 2019

Keywords: mHealth, healthy behaviors, worksite, university

Health care spending per person has risen in the United States for as long as expenditures have been tracked, yet population health appears to be deteriorating. In 2016, healthcare spending increased 4.3\% in the United States, reaching \$3.3 trillion, but life expectancy declined for the second consecutive year, which has not happened since 1963 (Centers for Medicare and Medical Services, 2018). A majority of chronic diseases can be prevented through health promotion and healthy lifestyle behaviors (O'Donnell, 2016). Up to half of all premature deaths in the U.S. are due to modifiable behavioral factors, including unhealthy eating, inactivity, and tobacco use (Institute of Medicine and National Research Council, 2015). Further, rising healthcare costs are widely regarded as unacceptable. Americans are living longer and desire a high quality of life (Stewart, Cutler, \& Rosen, 2013). These factors combined create an environment in which healthcare technology that helps people focus on wellness and assist them with healthy lifestyle behavior change is both desired by individuals and critical in controlling healthcare costs. 
Mobile applications (apps) are transforming the way we live. As of 2017, 3.6 billion mHealth apps were downloaded by consumers (Research2Guidance, 2018). Mobile health (mHealth) apps are health-related applications that aim to improve patients' health though multiple different functionalities and designs. There are currently more than 325,000 mHealth apps (including free and paid) publicly available in major app stores (Research2Guidance, 2018). These apps are widely used in healthcare practice in several fields, including chronic disease, such as diabetes mellitus, or the monitoring of weight loss in obese subjects. Most of the popular apps for health care and fitness aim to provide people with services to cover a wide range of functionalities, including self-monitoring to improve physical activity, the measurement of health and fitness parameters, determination of the Body Mass Index (BMI), the setting of weight goals, advice on nutrition and diet, mental well-being, and the monitoring of sleep and heart rate (Sannino \& De Pietro, 2011; Sannino, Forastiere \& De Pietro, 2017).

Results are mixed regarding the efficacy of wellness management apps. In a review of apps, Rivera and colleagues (2016) found that commercial mHealth apps for weight management lack important evidence-based features, do not involve healthcare experts in the development process and have not undergone rigorous scientific testing. Two systemic reviews revealed an overall advantage to using these apps, but also called for more high-quality controlled trials to test the efficacy of specific app features to distinguish effective from ineffective components (DiFilippo, Huang, Andrade, \& Chapman-Novakofski, 2015; Schoeppe et al., 2016).

The purpose of this pilot study was to test the feasibility, acceptability, and preliminary efficacy of the Fan Health Network (FHN) healthy behavior lifestyles application (app) on university faculty and staff's healthy lifestyle beliefs, healthy lifestyle behaviors, perceived stress and health outcomes.

\section{METHODS}

The current study took place at a large, mid-western, public land grant university. The study was approved by the university's institutional review board.

\section{Design}

A pre-experimental pre-test, post-test one group design was used to test the feasibility, acceptability and preliminary efficacy of the FHN healthy lifestyles app. Participants completed surveys at pretest, immediately following the intervention (posttest), and three months post-intervention. The intervention was eight weeks in duration, excluding data collection and recruitment.

\section{Sample}

The eligible study population included university faculty and staff, full and part-time, from the main and regional campuses as well as extension offices across the state. A message in the daily university-wide email system, which reaches close to 40,000 employees, was sent out one time. Additionally, targeted emails were sent out to specific employees on campus, including the wellness champion team (Amaya, Melnyk, Buffington, \& Battista, 2017), participants in previous wellness events, and to faculty and staff located in the health sciences colleges. Email invitations were sent to 3,065 employees, with an open rate of 1,570 (51\%). A total of 425 completed the preintervention survey; 51 participants of the original sample $(12 \%)$ completed the post-intervention survey; 39 participants of the immediate post-test sample completed the three month follow-up survey $(76 \%)$. 


\section{Measurement}

Healthy Lifestyle Beliefs Scale (HLBS). The HLBS is a 16-item instrument that was adapted from other Beliefs scales used in multiple prior studies (Melnyk \& Small, 2006). This scale taps beliefs about various facets of maintaining a healthy lifestyle (e.g., "I believe that I can be more active" and "I am sure that I will do what is best to lead a healthy life"). Subjects respond to each item on a Likert scale that ranges from 1 (strongly disagree) to 5 (strongly agree). Face validity was established with 10 adolescents and 10 adult parents. Content validity was established by eight specialists. Construct validity of the scale was supported through factor analysis. Cronbach alphas in preliminary studies have consistently been above .87 .

Healthy Lifestyle Behaviors. Healthy lifestyle behaviors were measured with the Healthy Lifestyle Behaviors Scale (Melnyk \& Small, 2006). Subjects respond to each of the 16 items (e.g., I exercised regularly; I talked about my worries or stressors, I made choices that lead to a healthy lifestyle) on a 5-point Likert scale that ranges from 1 (strongly disagree) to 5 (strongly agree). Face validity was established with 10 adolescents and adult parents and eight health experts established content validity. Construct validity has been supported through factor analysis from data obtained in previous studies. Cronbach alphas with teens and adult parents in prior pilot studies have been .80 and above.

Perceived Stress scale (PSS): The PSS (Cohen, Kamarck, \& Mermelstein, 1983) is the most widely used instrument for measuring the perception of stress in one's life. This 10 item instrument was designed to determine how unpredictable, overwhelming and uncontrollable they find their lives. Participants respond to each item on a Likert scale that ranges from 0 (never) to 4 (very often).

Blood Chemistry \& Anthropometric Measures (Self-Report): Participants self-reported (a) height, (b) weight, (c) waist circumference, (d) blood pressure and pulse, (e) total cholesterol, (f) HDL/non-HDL, and (g) Hbg A1c. BMI was calculated using the self-reported height and weight.

Demographic variables: Demographic data was collected, including: (a) age, (b) gender, (c) race/ethnicity, (d) educational level, (e) work unit type (f) worked per week, (g) marital status.

Feasibility and Acceptability was measured at the immediate post-test follow-up in the post-intervention survey. Questions included the app's usefulness, helpfulness and motivational components, on a scale of 1 (not useful/helpful) to 5 (extremely helpful/ useful). Items included in-app activities, such as postings, the huddle, athlete social interaction, tracking behavior and earning points. Additional open-ended questions provided the investigators with anecdotal participant feedback.

\section{Intervention}

The intervention components, from here forward referred to as the "challenge" were conducted within the FHN app. Participants downloaded the app from their smart phone app store, logged in using their employee credentials, and identified the starting point within the app for the "challenge." It was important for employees to identify themselves according to their employee credentials so that the program administrators were able to identify and approve the user as part of the pilot study, for data and engagement purposes.

Participants interacted with the engagement strategies provided by the FHN solution. The app components are built on the construct of self-regulation, which incorporates self-monitoring, feedback, and the enlistment of social support. Self-regulation is a well-documented, viable strategy to aid in healthy behavior change (Bandura, 1977, 1986, 1991, 1994). In the app, participants are able to follow notable professional sports figures ("Captains"), including alumni members of 
the university athletic community, current and past NFL® players, and other professional athletes and sports celebrities. Participants tracked and posted their exercise and nutrition behaviors in the app. They were included in social "huddles" with access to status updates, workouts and motivations from their Captain(s). Participants had access to weekly healthrelated articles on topics such as mental well-being, exercise and nutrition. They also completed weekly "quizzes" on the health related articles. The purpose of the quizzes was to further educate participants on the topics, and determine whether knowledge improved through the educational objectives set by the investigators. All intervention activities except for the surveys were accessed within the app. Additionally, based on their engagement with in-app activities, participants earned points to qualify for incentives based on scoring ranges. The uniqueness of the FHN app is the ability for participants to interact with role models and professional sports figures who may be dealing with similar health issues, particularly if they are not professional athletes anymore, or are trying to balance life, work and self-care.

\section{Analysis}

Feasibility and acceptability were analyzed by using the average score among participant responses. Open ended responses were collected and themed by topic. We further compared the pre-, immediate post-, and follow-up intervention surveys on measures of healthy lifestyle beliefs, healthy lifestyle behavior, and health indicators (perceived stress, blood pressure, BMI, waist circumference, pulse, cholesterol, and Hgb A1c) among participants responded to both the pre- and postintervention, and follow-up surveys using descriptive statistics and paired $t$-tests. $P$-values and effect sizes of the pre- to post -intervention changes were calculated. All tests were two-sided with a significance level of.05.

\section{RESULTS}

Four hundred twenty-five faculty and staff completed the pre-intervention survey. Of those, 236 registered for the challenge. Registering for the challenge means they downloaded the app on their smartphone or device and registered for the challenge in the app using their university-sponsored email address. One hundred fifty-five (66\%) participants that registered for the challenge logged some type of activity within the challenge. Among the 425 participants who responded to the pre-intervention survey, $51(12 \%)$ completed the post-intervention survey and $39(76 \%)$ completed the three month follow-up survey. A majority of participants at post-test were female (68.6\%), white (90.2\%), with a college degree $(39.2 \%)$, and married $(60.8 \%)$. The average age of participants was 45 years of age. A majority of participants were staff $(91 \%)$ and worked full-time $(96 \%)$.

There were positive changes in multiple outcome variables from pre- to immediate post-intervention. Healthy beliefs and healthy lifestyle behaviors increased, with significant improvement in healthy behaviors (mean \pm SD: 62.8 \pm 7.2 at pre- vs. $65.8 \pm 6.8$ at post-intervention, $p=.002)$. The improvement in healthy behaviors reached a near moderate effect size (Cohen's $d=.47$ ). Perceived stress significantly decreased throughout the intervention (11.9 \pm 6.6 at pre- vs. $10.5 \pm 6.2$ at post-intervention, $p=.03$ ) with a small effect size (Cohen's $d=.34$ ). Self-reported diastolic blood pressure, total cholesterol, non-HDL and HA1C decreased. Systolic blood pressure, BMI, waist circumference, and pulse remained relatively stable with very small decreases at post-test. Healthy beliefs, systolic and diastolic blood pressure, HDP waist circumference and Hgb A1C had small effects (Cohen's $d=.25-.34$ ). HDL increased (Table 1). 
Table 1

Pre-Test to Immediate Post-Test Changes in Outcomes

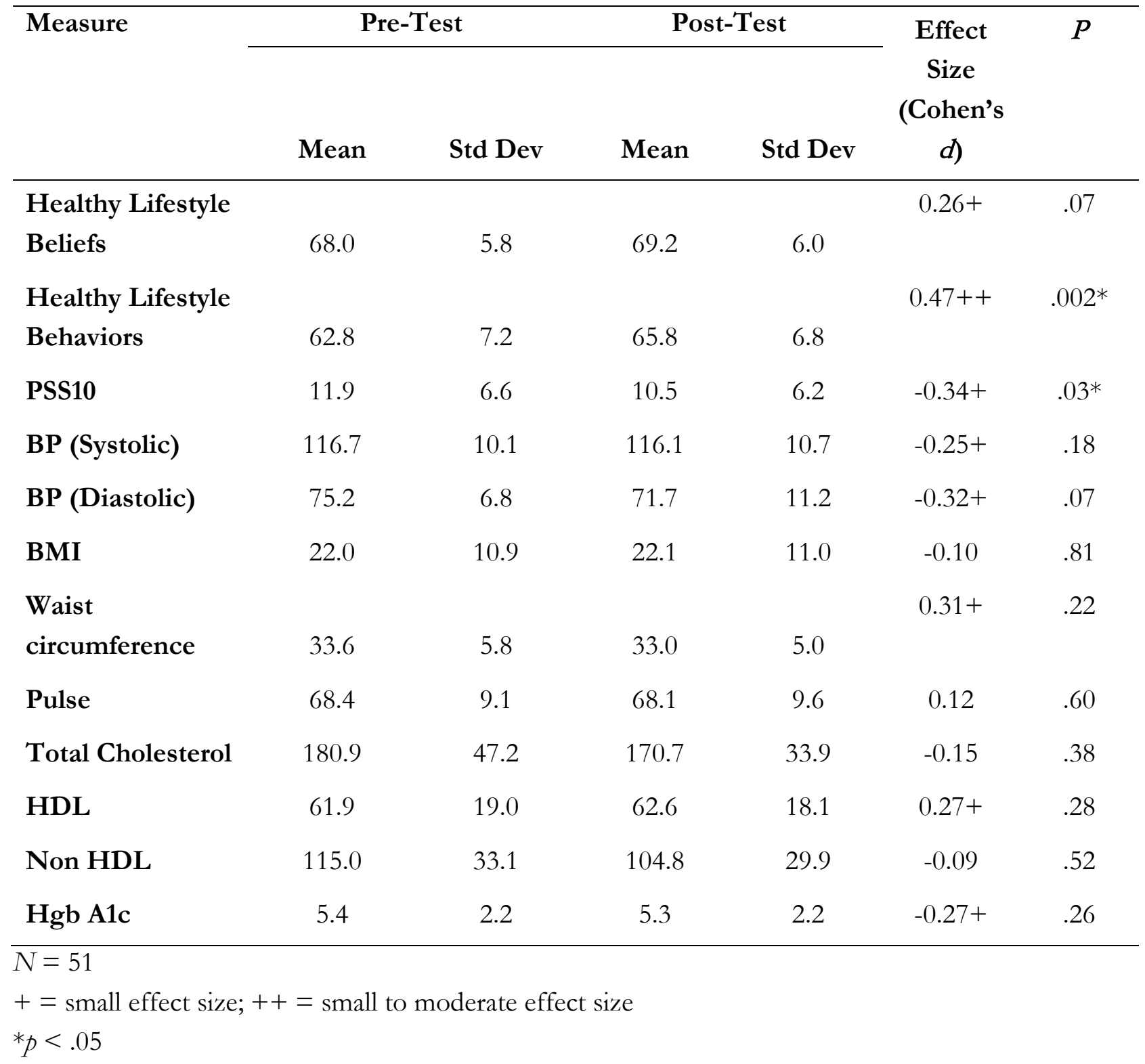

At the three month post-intervention follow-up, additional improvements were observed for body mass index (mean $\pm S D: 104.8 \pm 29.9$ vs. $21 \pm 10.7$ at post-intervention) and waist circumference (mean $\pm S D: 32.3 \pm 4.1$ vs. 33 \pm 5 at post-intervention). Healthy beliefs, perceived stress, systolic blood pressure, HDL and Hgb A1C remained steady. Healthy behaviors decreased slightly; diastolic blood pressure, total cholesterol and non-HDL increased slightly (Table 2). 
Table 2

Pre-Test to 3-Month Follow-Up Changes in FHN Study Outcomes

\begin{tabular}{lcccccc}
\hline Measure & \multicolumn{2}{c}{ Post-Test } & \multicolumn{2}{c}{ 3-Month Follow-Up } & $\begin{array}{c}\text { Effect Size } \\
\text { (Cohen's } \boldsymbol{d} \text { ) }\end{array}$ & $P$ \\
\cline { 2 - 5 } Hean & Std Dev & Mean & Std Dev & & \\
Beliefs & 69.2 & 6.0 & 69.4 & 7.4 & $0.24+$ & .14 \\
Healthy Lifestyle & 65.8 & 6.8 & 64.8 & 6.5 & $0.24+$ & .21 \\
Behaviors & & & & & & \\
PSS10 & 10.5 & 6.2 & 10.7 & 6.8 & -0.18 & .30 \\
BP (Systolic) & 116.1 & 10.7 & 116.7 & 14.5 & -0.11 & .92 \\
BP (Diastolic) & 71.7 & 11.2 & 73.6 & 7.0 & -0.08 & .38 \\
BMI & 22.1 & 11.0 & 21.0 & 10.7 & -0.02 & .62 \\
Waist circumference & 33.0 & 5.0 & 32.3 & 4.1 & -0.13 & .65 \\
Pulse & 68.1 & 9.6 & 67.8 & 11.8 & 0.19 & .38 \\
Total Cholesterol & 170.7 & 33.9 & 177.9 & 55.6 & $0.32+$ & .45 \\
HDL & 62.6 & 18.1 & 62.5 & 22.0 & -0.03 & .85 \\
Non HDL & 104.8 & 29.9 & 111.4 & 41.7 & $0.38+$ & .40 \\
Hgb A1c & 5.3 & 2.2 & 5.5 & 2.2 & $-0.26+$ & .43 \\
\hline
\end{tabular}

$n=39$

$+=$ small effect size

To meet educational objectives, participants were asked to complete "quizzes" throughout the intervention. Quizzes were related to a weekly in-app article on exercise, healthy eating or mental well-being. Eight articles and corresponding quizzes were disseminated over the intervention. The goal was for the majority of respondents to achieve a $75 \%$ or better on the quiz, which include 3 or 4 questions on the corresponding article. Six out of eight quiz results met the $75 \%$ goal. The two quizzes that did not meet the $75 \%$ criteria included an article on physical activity and one on the benefits of mindfulness. See Table 3 below for quiz outcomes.

Table 3

FHN Weekly Quiz Results

\begin{tabular}{rcc}
\hline Week & Number of Respondents & $\mathbf{7 5 \%}$ Minimum Threshold \\
\hline 1 & 67 & $66(98 \%)$ \\
2 & 73 & $72(98 \%)$ \\
3 & 54 & $54(100 \%)$ \\
4 & 57 & $41(72 \%)$ \\
5 & 45 & $45(100 \%)$ \\
6 & 41 & $27(66 \%)$ \\
7 & 20 & $19(98 \%)$ \\
8 & 21 & $19(90 \%)$ \\
\hline
\end{tabular}


Scores for feasibility and acceptability of the program that resulted in a 3.0 and above included usefulness of the app for obtaining recommended levels of moderate (3.7) and vigorous (3.6) physical activity, usefulness of the wellness articles (3.2) and earning incentive points (3.0). The app was motivating for earning incentive points (3.3). Scores 2.9 and below included usefulness for posting pictures of healthy meals (2.1), usefulness of the huddle (2.1), and motivation for interacting in the huddle (2.3). Based on open-ended responses, a majority of participants found the workout function $(n=18)$ the most motivating, while a majority chose "other" $(n=20)$ for aspects the least motivating, with a variety of comments. Fifteen participants cited the wellness articles as the most helpful aspect to the app, while eleven participants cited posting pictures and not able to connect a tracker (14) the least helpful.

\section{DISCUSSION}

Findings from the study indicate the FHN platform may be an effective strategy to improve the health of participants, as noted by the positive outcomes observed in the present study. Participants enhanced self-regulation skills, a critical component of behavior change (Bandura, 1991), through tracking healthy eating and physical activity in the challenge. By utilizing the power of social connections, engaging daily with athletes and fellow university colleagues (users), feedback was provided to individuals throughout the intervention. This attribute encouraged and motivated participants to continue adhering to their healthy lifestyle behaviors. With the positive results observed in this pilot study, on key physical and mental behaviors and outcomes, the self-regulation framework demonstrated to be an appropriate foundation for a health promoting-related app.

There are several limitations to note in this pilot study, including the sample, study design and the use of selfreported data. The sample characteristics were homogeneous in that a majority of participants were female, Caucasian, and married. Most were university staff. There also was a large drop off rate from the number of participants who registered and logged activity as a part of the challenge and those that completed the immediate post-test and three month follow-up survey. Engagement in health promoting behaviors varies across time, and drop-out could be due to extraneous variables. Additionally, it is noted that further research is needed on the effectiveness of the app itself, to examine efficacy, dose-response and to conduct a process evaluation of the intervention components.

The results of this pilot study may be skewed because they were based on a small number of participant responses to the post-intervention and follow-up surveys. A small sample size lacks statistical power to detect changes in outcomes, which is the rationale for calculating effect sizes which are not dependent on sample size to detect intervention effects (Melnyk \& Morrison-Beedy, 2019). Additionally, the study used a one-group pre-post design, which threatens internal validity, concluding that the intervention led to changes in the outcomes, not other extraneous factors. A randomized controlled trial, with a sample size based on a power analysis, is needed to determine the efficacy of the FHN app. Additionally, internal validity threats include (1) history, events that may have occurred outside of the program that influenced participation; (2) repeated testing, where participants may be conditioned to know that they are being tested, so spacing survey administrations at baseline, immediately post-intervention and 3 months following the intervention helped to minimize this threat; and (3) mortality rates, to which participants were encouraged to complete the assessments at all the time frames, and the investigators used incentives to help motivate participation. Lastly, the data for this pilot project is based on self-report, which poses a limitation on the validity of the data, particularly the anthropometric results.

Despite rapid expansion of mHealth apps, there are still multiple limitations of mHealth apps in improving the health of consumers and ultimately, population health, including lack of regulatory supervision, limited evidence on 
efficacy, and privacy and security concerns (Kao \& Liebovitz, 2017). There exists a great potential for the evolution of these apps, and additional research on their effectiveness is needed. With future opportunities to continue to test and refine the FHN app, it has the potential to be adopted by worksites and organizations as a population health strategy to lower health care costs, improve healthy behaviors and mental well-being, and reduce the risk and prevalence of chronic conditions. 


\section{REFERENCES}

Amaya, M., Melnyk, B.M., Buffington, B., \& Battista, L. (2017). Workplace Wellness Champions: Lessons learned and implications for future programming. Building Healthy Academic Communities Journal, 1(1), 59-67. https://doi.org/10.18061/bhac.v1i1.5744

Bandura, A. (1977). Self-efficacy: Toward a unifying theory of behavioral change. Psychological Review, 84(2), $191-215$. https://doi.org/10.1037/0033-295X.84.2.191

Bandura, A. (1986). Social foundations of thought and action: A social cognitive theory. Englewood Cliffs, NJ: Prentice-Hall.

Bandura, A. (1991). Social cognitive theory of self-regulation. Organizational Behavior and Human Decision Processes, 50 , 248-287. https://doi.org/10.1016/0749-5978(91)90022-L

Bandura, A. (1994). Social cognitive theory and exercise of control over HIV infection. In R. J.DiClemente and J. L. Peterson (Eds.), Preventing AIDS: Theories and methods of behavioral interventions (pp. 25-59). New York, NY: Plenum. https://doi.org/10.1007/978-1-4899-1193-3_3

Center for Medicare and Medical Services (2018). National Health Expenditures 2017 Highlights. Retrieved from https://www.cms.gov/Research-Statistics-Data-and-Systems/Statistics-Trends-and-

Reports/NationalHealthExpendData/Downloads/highlights.pdf

Cohen, S., Kamarck, T., \& Mermelstein, R. (1983). A global measure of perceived stress. Journal of Health and Social Behavior, 24, 385-396. https://doi.org/10.2307/2136404

DiFilippo, K.N., Huang, W.-H., Andrade, J. E., \& Chapman-Novakofski, K.M. (2015). The use of mobile apps to improve nutrition outcomes: A systematic literature review. Journal of Telemedicine and Telecare, 21(5), $243-253$. https://doi.org/10.1177/1357633X15572203

Kao, C.K. \& Liebovitz, D.M. (2017). Consumer mobile health apps: current state, barriers, and future directions. American Academy of Physical Medicine and Rehabilitation, 9(5S), S106-S115. https://doi.org/10.1016/j.pmrj.2017.02.018

Melnyk, B.M., \& Morrison-Beedy, D. (Eds.). (2019). Intervention Research and Evidence-Based Quality Improvement: Designing, Conducting, Analyzing and Funding, A Practical Guide for Success (2nd ed.) New York, NY: Springer Publishing Company. https://doi.org/10.1891/9780826155719

Melnyk, B. M., \& Small, L. (2006). Mental health correlates of healthy lifestyle attitudes, beliefs, choices, and behaviors in overweight adolescents. Journal of Pediatric Health Care, 20(6). 401-406. https://doi.org/10.1016/j.pedhc.2006.03.004

Institute of Medicine and National Research Council (2015). Measuring the Risks and Causes of Premature Death: Summary of Workshops. Washington, DC: The National Academies Press. https://doi.org/10.17226/21656

O’Donnell, M.P. (2016). Health Promotion in the Workplace ( $5^{\text {th }}$ ed.). Troy, MI: Art \& Science of Health Promotion Institute.

Research2Guidance (2018). Mobile health market report 2017-2018. Retrieved from www.research2guidance.com 
Rivera J., McPherson, A., Hamilton, J., Birken, C., Coons, M., Iyer, A., Lalloo, C., Stinson, J. (2016). Mobile apps for weight management: a scoping review. JMIR MHealth and UHealth, 4(3), e87.

https://doi.org/10.2196/mhealth.5115

Sannino, G., \& De Pietro G. (2011). An evolved ehealth monitoring system for a nuclear medicine department. Developments in E-systems Engineering, 3-6. https://doi.org/10.1109/DeSE.2011.67

Sannino, G., Forastiere, M., \& De Pietro, G. (2017). A wellness mobile app for smart health: Pilot study design and results. Sensors, 17(3), 611. https://doi.org/10.3390/s17030611

Schoeppe, S., Alley, S., Van Lippevelde, W., Bray, N., Williams, S.L., Duncan, M.J., \& Vandelanotte, C. (2016). Efficacy of interventions that use apps to improve diet, physical activity and sedentary behaviour: A systematic review. International Journal of Behavioral Nutrition and Physical Activity, 13, 127. https://doi.org/10.1186/s12966-016-0454-y

Stewart, S. T., Cutler, D. M., \& Rosen, A. B. (2013). US trends in quality adjusted life expectancy from 1987 to 2008: Combining national surveys to more broadly track the health of the nation. American Journal of Public Health, 103, e78-e87. https://doi.org/10.2105/AJPH.2013.301250

Author correspondent may be addressed to:

Megan Amaya, PhD, CHES

Assistant Professor of Clinical Nursing

The Ohio State University College of Nursing

1585 Neil Avenue

Columbus, OH 43210

amaya.13@osu.edu 